tika vor Knochenfrakturen postuliert. Die jetzige Studie hat besonderes Gewicht wegen der großen Patientenzahl, der langen Studiendauer und der Randomisierung auf drei Antihypertensiva. Da es keinen Placeboarm gibt, kann nicht entschieden werden, ob Antihypertensiva, z. B. durch zu starke Blutdrucksenkung, das Sturz- und Frakturrisiko erhöhen. Es gibt aber inzwischen zahlreiche Studien, nach denen di- verse kardiovaskuläre Medikamente einschließlich Antihypertensiva das Frakturrisiko nicht erhöhten, sodass es sich um einen Schutzeffekt der Diuretika handeln muss. Der wahrscheinliche Mechanismus: Thiaziddiuretika verringern die renale Kalziumausscheidung. Deshalb galten sie lange als erste Wahl für die Behandlung von kalziumhaltigen Nierensteinen bei Hyperkalzurie. Bei Hyperkalzämie (z.B. Hyper- parathyreoidismus, Knochenmetastasen) sind sie deswegen kontraindiziert.

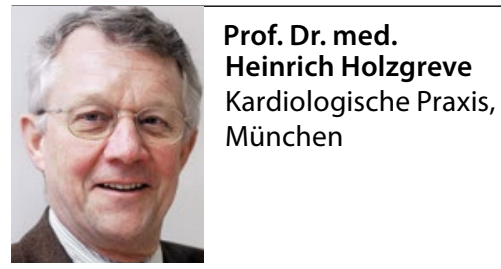

\title{
Fitnessarmbänder messen den Puls unter Belastung ungenau
}

\section{Der Verkauf von Fitnesstrackern ist in den letzten Jahren explodiert. Aller- dings haben schon frühere, auch in Deutschland durchgeführte Tests gezeigt, dass die Herzfrequenzmessung mit diesen Armbändern oft ungenau ist.}

ᄃ itnessarmbänder zählen Schritte, erFechnen den Kalorienverbrauch und messen die Herzfrequenz. Selbst der

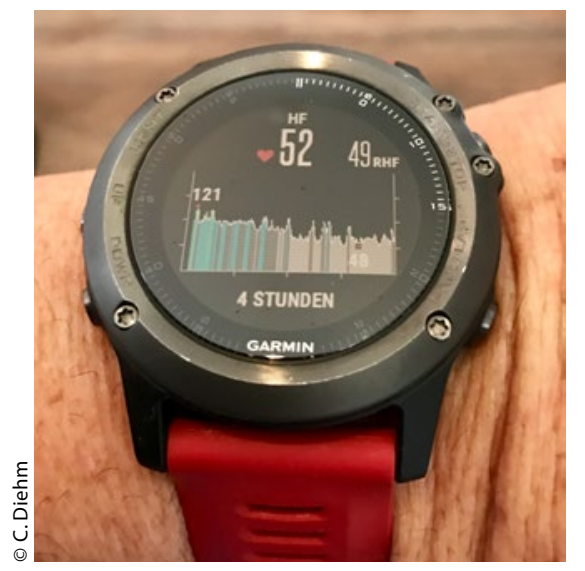

Die Pulsmessung über einen Brustgurt ist genauer als am Handgelgenk.
Schlafrhythmus kann dokumentiert werden. Fitnessarmbänder sollen ihre Träger motivieren, aktiver und gesünder zu leben. In Verbindung mit einer App können die Daten in Smartphones und in Computer ausgelesen werden. Die meisten modernen Tracker sind mit GPS ausgestattet.

Sportmediziner und Kardiologen der Cleveland Clinic (Ohio) haben nun die Genauigkeit von Fitnessarmbändern und Pulsuhren in Ruhe und unter Belastung (Laufband) mit und ohne Brustgurt bei Gesunden untersucht. Der Vergleich erfolgte gegen ein konventionelles EKG bzw. ein Belastungs-EKG.

Untersucht wurden 50 Probanden, $58 \%$ waren Frauen. 14\% waren Afroamerikaner. Gemessen wurde in Ruhe und bei Laufbandgeschwindigkeiten von 2, 3, 4 und 6 Meilen pro Stunde. Pa-

\begin{tabular}{|l|l|}
\hline \multicolumn{2}{|l}{ Tab. 1: Genauigkeit von Fitnessarmbändern } \\
\hline Typ und Hersteller & Konkordanz-Korrelationskoeffizient (95\%-KI) \\
\hline Apple Watch (Apple) & $0,91(0,884-0,929)$ \\
\hline Mio Fuse (Mio Global) & $0,91(0,882-0,929)$ \\
\hline Fitbit Charge HR (Fitbit) & $0,84(0,791-0,872)$ \\
\hline Basis Peak (Basis) & $0,83(0,779-0,865)$ \\
\hline Polar H7 (Polar USA)* & $0,99(0,987-0,991)$ \\
\hline${ }^{*}$ mit Brustgurt & \\
\hline
\end{tabular}

tienten mit Herzschrittmacher und manifesten Herzerkrankungen wurden von der Studie ausgeschlossen. Probanden, die Medikamente einnahmen, die den Herzrhythmus beeinflussen, wurden ebenfalls von der Studie ausgeschlossen. Der mittlere Body-Mass-Index lag bei $23,5 \mathrm{~kg} / \mathrm{m}^{2}$.

Die Ergebnisse der Fitnessarmbänder waren in Ruhe am besten. Unter Belastung allerdings wurden die Messwerte sehr ungenau (Tab. 1). Statistisch gesehen war jede fünfte Messung sehr ungenau. Fitnessarmbänder messen mit optischen Sensoren, die die Gewebsdurchblutung lokal evaluieren. Vor allem bei höheren Pulsfrequenzen können die Messwerte dadurch ungenau werden. Bei der Apple Watch lagen bei den jungen gesunden Erwachsenen $95 \%$ der gemessenen Werte plus minus 25 Schläge vom realen Wert entfernt - wenn ohne Brustgurt gemessen wurde.

Wang R et al. Accuracy of wrist-worn heart rate monitors. JAMA Cardiol. 2017;2(1):104-6

\section{Kommentar}

Wer sich eine solche Pulsuhr bzw. ein Fitnessarmband anschafft - egal ob fürs Training oder auf Empfehlung eines Kardiologen bei einer Herzerkrankung - ist gut beraten, wenn er sich zusätzlich einen Brustgurt besorgt und diesen so oft wie möglich anlegt.

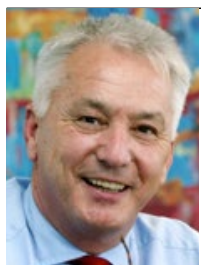

Prof. Dr. med. Curt Diehm Max-Grundig-Klinik, Bühl 